\title{
Bezafibrate treatment is associated with a reduced rate of re-hospitalization in smokers after acute coronary syndrome
}

\author{
Robert Klempfner, 2, ${ }^{1,}$, Ilan Goldenberg', 2,3,4 ${ }^{\text {Enrique Z. Fisman }}{ }^{5}$, Uri Amit ${ }^{3}$, \\ Alexander Haitovich ${ }^{3}$, Shlomi Matetzky ${ }^{2,3}$, Diego Medvedofsky $^{2}$, \\ Joseph Shemesh ${ }^{1}$, Alexander Tenenbaum ${ }^{1,2,3,5}$ \\ ${ }^{1}$ Cardiac Rehabilitation Institute, Sackler Faculty of Medicine, Tel-Aviv, Israel \\ ${ }^{2}$ Leviev Heart Institute, Sackler Faculty of Medicine, Tel-Aviv, Israel \\ ${ }^{3}$ Israeli Society for the Prevention of Heart Attacks, Chaim Sheba Medical Center, \\ Tel-Hashomer, Sackler Faculty of Medicine, Tel-Aviv University, Tel-Aviv, Israel \\ ${ }^{4}$ Heart Research Follow-up Program, University of Rochester Medical Center, \\ Rochester, New York, United States \\ ${ }^{5}$ Cardiovascular Diabetology Research Foundation, Holon, Israel
}

\begin{abstract}
Background: Significantly increased rate of hospitalizations in current smokers is a major smoking-related problem which is associated with a heavy economic burden, whereas cardiovascular disease accounted for nearly half of hospitalizations. The effect of bezafibrate on the rate of re-hospitalization in smokers already treated with statin immediately post-acute coronary syndrome (ACS) is unknown. The aim of this study was to investigate 30-day rate of re-hospitalization in current smokers participating in the ACS Israeli Surveys (ACSIS) and who were treated on discharge with a bezafibrate/statin combination vs. statin alone.
\end{abstract}

Methods: The study population comprised 3392 patients with confirmed current smoking status from the ACSIS 2000, 2002, 2004, 2006, 2008 and 2010 enrollment waves who were alive on discharge and received statin. Of these, 3189 (94\%) were discharged with statin alone, $203(6 \%)$ with a combination of a statin and bezafibrate.

Results: Thirty-day re-hospitalization rate was significantly lower in patients from the combination group than in their counterparts from the statin monotherapy group: $12.8 \% \mathrm{vs.} 19 \%$, $p=0.028$. Multivariable analysis identified the combined bezafibrate/statin treatment as an independent predictor of reduced risk of 30-day re-hospitalization rate with odds ratio (OR) 0.53 (95\% confidence interval [CI] 0.31-0.91), and it corresponded to $47 \%$ risk reduction. Other significant variables in our model associated with independent risk of 30-day re-hospitalization rate during the follow-up were female gender (OR 1.43, CI 1.05-1.95, $p=0.03)$ and age > 65 years (OR 1.49, CI 1.13-1.95, $p=0.004)$.

Conclusions: Adding bezafibrate to statin in smokers was associated with a significantly reduced 30-day rate of re-hospitalization after ACS. (Cardiol J 2014; 21, 4: 364-369)

Key words: acute coronary syndrome, bezafibrate, smoking

Address for correspondence: Prof. Alexander Tenenbaum, Cardiac Rehabilitation Institute, Chaim Sheba Medical Center, Tel-Hashomer, 52621 Israel, tel: 972-3-5302578, fax: 972-3-5303084, e-mail: altenen@yahoo.com 


\section{Introduction}

Tobacco use is a principal contributor to the development of coronary artery disease (CAD) and its consequences, including sudden cardiac death, acute myocardial infarction (MI), heart failure and increased rate of hospitalizations. Significantly increased rate of hospitalizations in current smokers is a major smoking-related problem which is associated with heavy economic burden, whereas cardiovascular disease (CVD) accounted for nearly half of hospitalizations [1-3].

Smoking leads to oxidative stress, a hypercoagulable and pro-inflammatory states which are strongly associated with atherothrombosis and CAD [4-10]. In the INTERHEART study, tobacco use was associated with a 3 -fold increase in the risk for nonfatal acute MI [2]. The relationship was dose-dependent, which showed that those who smoked a pack per day or more had a 4.6 -fold increase in the risk compared to non-smokers [3].

Bezafibrate is a pharmacological ligand of all peroxisome proliferators-activated receptors (PPARs) with ability to increase high density lipoprotein-cholesterol (HDL-C) and decrease triglyceride, glucose and fibrinogen concentrations [11-19]. The effect of bezafibrate on the rate of re-hospitalizations in smokers already treated by statin immediately post-acute coronary syndrome (ACS) is unknown.

The main aim of this study was to investigate 30-day rate of re-hospitalization in current smokers participating in the ACS Israeli Surveys (ACSIS) who were treated on discharge with a bezafibrate/ /statin combination vs. statin alone. In addition, 30-day rate of major adverse cardiovascular events (MACE) and 1-year mortality rate was also evaluated.

\section{Methods}

\section{Study population}

Our patients have been drawn from the ACSIS 2000, 2002, 2004, 2006, 2008 and 2010 enrollment waves. Details of the ACSIS Registry have been previously reported [20].

In brief, the ACSIS Registry is a 2-month nationwide survey conducted biennially that prospectively collects data from all ACS admissions in all 25 coronary care units (CCU) in Israel. Patient management was at the discretion of the attending physicians. Eligibility for the study was validated before discharge from the CCU. Discharge diagnoses were recorded as determined by the attending physicians based on clinical, electrocardiographic, and enzymatic criteria. Demographic, historical and clinical data, including medical management, were recorded on pre-specified forms by dedicated study physicians. The Central Data Coordinating Center (based at the Sheba Medical Center) was responsible for the collection of all case report forms and the Israel Heart Society was responsible for keeping the survey database. Thirty-day outcome rates and 1-year mortality rate were ascertained by hospital chart review, telephone contact and use of the Israeli National Population Registry.

Our study population comprised 3392 patients who met the following criteria: were alive on discharge from the hospital, received statin, 30-day MACE rate was available, bezafibrate treatment on discharge was recorded, current smoking status - as registered on hospital admission. Of these, 3189 (94\%) were discharged with statin alone, 203 $(6 \%)$ with a combination of a statin and bezafibrate.

\section{Ethics statement}

This register-based analysis of pre-existing data was conducted according to the principles expressed in the Declaration of Helsinki. The ACSIS was approved by the ethics committee of the Sheba Medical Center. All patients provided written informed consent for the collection of data and subsequent analysis.

\section{Endpoints and definitions}

In our study we used pre-specified definitions of the ACSIS. The diagnosis of diabetes was done by the attending physician based on the reported history, medical records and/or for patients with fasting blood glucose of $\geq 126 \mathrm{mg} / \mathrm{dL}(7 \mathrm{mmol} / \mathrm{L})$ registered twice or taking any type of pharmacologic anti-diabetic treatment prior to enrollment. Smoking habits were determined on the basis of self-reporting by the patients during interviews held with study physicians. The diagnosis of hypertension was done based on the reported history, medical records and/or for patients with blood pressure $>140 / 90 \mathrm{~mm} \mathrm{Hg}$ registered twice, or treatment with antihypertensive drugs prior to enrollment.

The primary endpoint of our study was 30-day re-hospitalization rate. Secondary endpoints were 30-day MACE rate (which was defined as a composite measure of 30-day all-cause mortality, recurrent MI, recurrent ischemia, stent thrombosis, ischemic stroke and urgent revascularization) and 1-year all-cause mortality. 
Table 1. Baseline characteristics and co-morbidities of the study population.

\begin{tabular}{lccc}
\hline Characteristics & Statin alone $(\mathbf{n}=\mathbf{3 1 8 9})$ & Bezafibrate/Statin $(\mathbf{n}=\mathbf{2 0 3})$ & $\mathbf{P}$ \\
\hline Age $[$ years] & $55.8 \pm 10.3$ & $52.5 \pm 9.7$ & $<0.0001$ \\
Body mass index $\left[\mathrm{kg} / \mathrm{m}^{2}\right]$ & $27.1 \pm 4.2$ & $29.3 \pm 4.4$ & $<0.0001$ \\
Weight $[\mathrm{kg}]$ & $80.2 \pm 14.5$ & $86.7 \pm 15.5$ & $<0.0001$ \\
Women & $352(11 \%)$ & $25(12.3 \%)$ & 0.567 \\
Hypertension & $1333(41.8 \%)$ & $116(57.1 \%)$ & $<0.0001$ \\
Diabetes & $786(24.6 \%)$ & $80(39.4 \%)$ & $<0.0001$ \\
History of stroke & $169(5.3 \%)$ & $13(6.4 \%)$ & 0.498 \\
Chronic renal failure & $144(4.5 \%)$ & $9(4.4 \%)$ & 0.956 \\
Peripheral vascular disease & $258(8.1 \%)$ & $11(5.4 \%)$ & 0.172 \\
\hline
\end{tabular}

Data are number (\%) of patients or mean \pm standard deviation.

Table 2. In-hospital cardiac interventions and laboratory values.

\begin{tabular}{lccc}
\hline Characteristics & Statin alone $(\mathbf{n}=\mathbf{3 1 8 9})$ & Bezafibrate/Statin $(\mathbf{n}=\mathbf{2 0 3})$ & $\mathbf{P}$ \\
\hline Coronary artery bypass graft & $131 / 3189(4.1 \%)$ & $7 / 203(3.4 \%)$ & 0.645 \\
Percutaneous coronary intervention & $1394 / 2866(48.6 \%)$ & $105 / 177(59.3 \%)$ & 0.006 \\
Only angiography & $398 / 2778(14.3 \%)$ & $36 / 179(20.1 \%)$ & 0.034 \\
Systolic blood pressure [mm Hg] & $139.07 \pm 27.725$ & $142.16 \pm 24.179$ & 0.122 \\
Diastolic blood pressure [mm Hg] & $82.25 \pm 16.979$ & $84.92 \pm 15.740$ & 0.030 \\
Heart rate [bpm] & $79.58 \pm 18.980$ & $80.05 \pm 14.924$ & 0.727 \\
Glucose $[\mathrm{mg} / \mathrm{dL}]$ & $140.30 \pm 67.184$ & $155.00 \pm 77.210$ & 0.008 \\
Total cholesterol [mg/dL] & $197.78 \pm 45.992$ & $213.64 \pm 48.884$ & $<0.0001$ \\
HDL-cholesterol [mg/dL] & $39.02 \pm 11.716$ & $32.86 \pm 6.979$ & $<0.0001$ \\
LDL-cholesterol [mg/dL] & $122.00 \pm 38.677$ & $118.30 \pm 43.048$ & 0.287 \\
Triglycerides [mg/dL] & $170.38 \pm 110.829$ & $396.182 \pm 80.008$ & $<0.0001$ \\
Creatinine $[\mathrm{mg} / \mathrm{dL}]$ & $1.05 \pm 0.591$ & $1.03 \pm 0.384$ & 0.523 \\
\hline
\end{tabular}

Data are number of patients/number of interventions/(\%) for interventions or mean \pm standard deviation for laboratory values.

\section{Statistical analysis}

Statistical analysis was performed using SPSS statistical software. Categorical variables were expressed as percentages, and continuous variables were expressed as mean \pm standard deviation. Comparisons of variables were performed by $\chi^{2}$ and Fisher's exact test for categorical variables and by unpaired ANOVA test for continuous variables. Survival curves were derived using the Kaplan-Meier approach, and unadjusted comparisons of survival curves were performed using the log-rank test.

In order to determine whether the combination of bezafibrate and statin vs. statin only treatment on discharge is an independent explanatory variable for re-hospitalization rate, multivariable logistic regression analysis was applied with adjustment for the following variables: age, gender, hypertension, diastolic blood pressure, diabetes, performing of the coronary angiography and percutaneous coronary intervention.

Results are presented as odds ratio (OR) with the appropriate $95 \%$ confidence interval (CI). All tests were 2 -sided and $p$ value $<0.05$ was considered statistically significant.

\section{Results}

Our population was categorized into 2 groups: 1) patients receiving on discharge statin monotherapy -3189 ; and 2) patients receiving on discharge a combined bezafibrate/statin treatment -203 .

\section{Baseline data}

The main clinical and laboratory characteristics of patients are presented in Tables 1 and 2 . The majority of patients in both groups were men. $\mathrm{Pa}-$ tients receiving bezafibrate were younger $(52.47 \pm$ \pm 9.731 vs. $55.84 \pm 10.3$ years). On the other hand, 
Table 3. Distribution of cardiovascular drugs among the study patients (on discharge).

\begin{tabular}{lccc}
\hline Drugs & $\begin{array}{c}\text { Statin alone } \\
\text { (n = 3189) }\end{array}$ & $\begin{array}{c}\text { Bezafibrate/Statin } \\
\text { (n = 203) }\end{array}$ & P \\
\hline Aspirin & $97.7 \%$ & $97.1 \%$ & 0.60 \\
Clopidogrel & $76.9 \%$ & $76.1 \%$ & 0.77 \\
Beta-blockers & $82.4 \%$ & $90.0 \%$ & 0.004 \\
Nitrates & $13.4 \%$ & $9.1 \%$ & 0.11 \\
Calcium channel blockers & $11.2 \%$ & $11.4 \%$ & 0.18 \\
Diuretics & $12.2 \%$ & $11.4 \%$ & 0.75 \\
Aldosterone antagonist & $4.4 \%$ & $7.5 \%$ & 0.06 \\
Angiotensin converting enzyme inhibitors & $71.2 \%$ & $73.3 \%$ & 0.51 \\
Angiotenesin receptor blockers & $4.3 \%$ & $7.9 \%$ & 0.14 \\
Insulin & $4.8 \%$ & $10.0 \%$ & $<01$ \\
\hline
\end{tabular}

Table 4. Outcomes of the study population during the follow-up (crude data).

\begin{tabular}{lccc}
\hline Outcomes & $\begin{array}{c}\text { Statin alone } \\
(\mathbf{n}=3189)\end{array}$ & $\begin{array}{c}\text { Fibrate/Statin } \\
(\mathbf{n}=203)\end{array}$ & $\mathbf{P}$ \\
\hline 30-day MACE & $328(10.3 \%)$ & $15(7.4 \%)$ & 0.187 \\
30-day re-hospitalization & $606(19 \%)$ & $26(12.8 \%)$ & 0.028 \\
1-year all-cause mortality & $105(3.3 \%)$ & $5(2.5 \%)$ & 0.527 \\
\hline
\end{tabular}

Data are number of events/(\%). The primary endpoints of this study were a) 30-day major adverse coronary events (MACE): all-cause mortality, recurrent myocardial infarction, recurrent ischemia, stent thrombosis, ischemic stroke, urgent revascularization during follow-up; b) 30-day re-hospitalization rate.

they had appreciably more co-morbidities (hypertension and diabetes) and unfavorable cardio-metabolic profile (as regards glucose, total cholesterol, triglyceride and HDL-C levels). Weight, body mass index and diastolic blood pressure were also significantly higher in patients receiving bezafibrate.

No significant differences between the groups were found for history of stroke, chronic renal failure and peripheral vascular disease. In patients receiving bezafibrate were performed more inhospital coronary angiographies and percutaneous coronary interventions.

Data regarding treatment with cardiovascular drugs among the study groups are presented in Table 3. Antiplatelet drugs (aspirin and clopidogrel), beta-blockers and angiotensin converting enzyme inhibitors were the most commonly used medications. The use of nitrates, calcium antagonists and diuretics was relatively low.

More patients from the combination bezafibrate/statin group received beta-blockers and insulin than their counterparts. There were no significant differences in the proportion of patients receiving other cardiovascular drugs.

\section{Outcomes of the study population during follow-up}

During the follow-up period of 30 days, development of MACE was recorded in 343 patients: in $328(10.3 \%)$ of patients from the statin monotherapy group vs. $15(7.4 \%)$ from the combined bezafibrate/statin group $(\mathrm{p}=0.19)$ (Table 4$)$.

Thirty-day re-hospitalization rate was significantly lower in patients from the combination group than in their counterparts from the statin monotherapy group: $12.8 \%$ vs. $19 \%(\mathrm{p}=0.028)$.

Crude 1-year mortality rates in patients of the combination bezafibrate/statin group tended to be lower than in their counterparts of the statin monotherapy group (2.5\% vs. $3.2 \%)$, but this trend did not reach statistical significance. Kaplan-Meier analysis showed that the cumulative probability of survival at 1-year of follow-up (in accordance with the time of occurrence) was numerically higher among patients who received combined bezafibrate/statin therapy compared with patients who received statin monotherapy. However, possibly due to sample size limitations and relatively low number of mortality events, this trend was statistically non-significant. 
Multivariable analysis identified the combined bezafibrate/statin treatment as an independent predictor of reduced risk of 30-day re-hospitalization rate with OR $0.53,95 \%$ CI $0.31-0.91$, corresponding to $47 \%$ risk reduction. Other significant variables in our model associated with independent risk of 30-day re-hospitalization rate during follow-up were female gender (OR 1.43, 95\% CI 1.05-1.95, $\mathrm{p}=0.03)$ and age $>65$ years (OR $1.49,95 \% \mathrm{CI}$ $1.13-1.95, \mathrm{p}=0.004)$.

\section{Discussion}

The main finding of our study is a significant association between addition of bezafibrate to statin in smokers after ACS and reduced 30-day rate of re-hospitalization.

Also 30-day MACE and 1-year mortality rates were numerically lower among patients who received combined bezafibrate/statin therapy compared with patients who received statin monotherapy. However, possibly due to sample size limitations and relatively low number of mortality events, this trend was statistically non-significant. Smoking status is strongly associated with admissions and duration of hospitalizations with large healthcare expenditures. A number of studies have found smoking to be associated with more hospital days and an increased utilization of hospital services [21-24], whereas CVD accounted for near half of hospitalizations $[1,25]$.

The economic consequences of tobacco use are both direct in the form of higher health care costs and transportation to and from health care facility, and indirect costs in the form of productivity losses due to morbidity and premature mortality.

Growing evidence supports the hypothesis that oxidative stress, glucose metabolism status, a hyper-coagulable and pro-inflammatory state, leading to atherothrombosis, provide a pathophysiological link between cigarette smoking and CAD [26-29]. Levels of circulating fibrinogen, one of the strongest predictors of coronary events, are increased in smokers [10,28]. Smoking may also enhance circulating levels of inflammation-associated factors such as the acute phase proteins-C-reactive protein (CRP), serum amyloid $\mathrm{A}$, complement proteins, interleukins (IL): IL-6, IL-2, IL-18, and tumor necrosis factor alpha (TNF- $\alpha$ ) $[7,26]$.

Bezafibrate is a pharmacological ligand of all PPARs with ability to decrease triglyceride, glucose levels, insulin resistance and increase HDL-C [11-13]. In addition, bezafibrate has important fibrinogen-related properties and anti-inflammatory effects: it lowers fibrinogen concentrations and reduces other markers of systemic inflammation like TNF- $\alpha$, IL-6 and CRP [14-19]. Therefore, a significant association between bezafibrate treatment and reduced 30-day rate of re-hospitalizations in smokers after ACS is biologically plausible.

\section{Limitations of the study}

Our analysis was not a randomized controlled trial and we cannot rule out other factors that could have influenced the observed improvements in clinical outcomes. Number of patients received statin and bezafibrate combination was relatively small compared to the group of statin alone. Therefore, caution should be taken in using our findings which require further confirmation in a prospective controlled clinical trial.

\section{Conclusions}

In conclusion, adding bezafibrate to statin in smokers was associated with a significantly reduced 30-day rate of re-hospitalization after ACS.

Conflict of interest: none declared

\section{References}

1. Anthonisen NR, Connett JE, Enright PL, Manfreda J. Hospitalizations and mortality in the Lung Health Study. Am J Respir Crit Care Med, 2002; 166: 333-339.

2. Yusuf S, Hawken S, Ounpuu S et al. Effect of potentially modifiable risk factors associated with myocardial infarction in 52 countries (the INTERHEART study): case-control study. Lancet, 2004; 364: 937-952.

3. Teo KK, Ounpuu S, Hawken S et al. Tobacco use and risk of myocardial infarction in 52 countries in the INTERHEART study: A case-control study. Lancet, 2006; 368: 647-658.

4. Csiszar A, Podlutsky A, Wolin MS, Losonczy G, Pacher P, UngvariZ. Oxidative stress and accelerated vascular aging: Implications for cigarette smoking. Front Biosci, 2009; 14: 128-144.

5. Bullen C. Impact of tobacco smoking and smoking cessation on cardiovascular risk and disease. Expert Rev Cardiovasc Ther, 2008; 6: 883-895.

6. Ernst E, Koenig W, Matrai A, Filipiak B, Stieber J. Blood rheology in healthy cigarette smokers. Arteriosclerosis, 1988; 8: 385-388.

7. Tonstad S, Cowan JL. C-reactive protein as a predictor of disease in smokers and former smokers: A review. Int J Clin Pract, 2009; 63: 1634-1641.

8. Wilhelmsen L. Coronary heart disease: Epidemiology of smoking and intervention studies of smoking. Am Heart J, 1988; 115: 242-249.

9. Benowitz NL. Pharmacology of nicotine: Addiction, smokinginduced disease, and therapeutics. Ann Rev Pharmacol Toxicol, 2009; 49: 57-71.

10. MacCallum PK. Markers of hemostasis and systemic inflammation in heart disease and atherosclerosis in smokers. Proc Am Thorac Soc, 2005; 2: 34-43. 
11. The BIP Study Group: Secondary prevention by raising HDL cholesterol and reducing triglycerides in patients with coronary artery disease: The Bezafibrate Infarction Prevention (BIP) study. Circulation, 2000; 102: 21-27.

12. Tenenbaum A, Fisman EZ. Fibrates are an essential part of modern anti-dyslipidemic arsenal: Spotlight on atherogenic dyslipidemia and residual risk reduction. Cardiovasc Diabetol, 2012; 11: 125.

13. Meade T, Zuhrie R, Cook C, Cooper J. Bezafibrate in men with lower extremity arterial disease: Randomised controlled trial. BMJ, 2002; 325: 1139.

14. Ikewaki K, Noma K, Tohyama J, Kido T, Mochizuki S. Effects of bezafibrate on lipoprotein subclasses and inflammatory markers in patients with hypertriglyceridemia: A nuclear magnetic resonance study. Int J Cardiol, 2005; 101: 441-447.

15. Jonkers IJ, Mohrschladt MF, Westendorp RG, van der Laarse A, Smelt AH. Severe hypertriglyceridemia with insulin resistance is associated with systemic inflammation: Reversal with bezafibrate therapy in a randomized controlled trial. Am J Med, 2002; 112: 275-280.

16. Teramoto T, Shirai K, Daida H, Yamada N. Effects of bezafibrate on lipid and glucose metabolism in dyslipidemic patients with diabetes: The J-BENEFIT study. Cardiovasc Diabetol, 2012; 11: 29.

17. Madrid-Miller A, Moreno-Ruiz LA, Borrayo-Sánchez G, AlmeidaGutiérrez E, Martínez-Gómez DF, Jáuregui-Aguilar R. Impact of bezafibrate treatment in patients with hyperfibrinogenemia and ST-elevation acute myocardial infarction: A randomized clinical trial. Cir Cir, 2010; 78: 229-237.

18. Tenenbaum A, Fisman EZ. Balanced pan-PPAR activator bezafibrate in combination with statin: Comprehensive lipids control and diabetes prevention? Cardiovasc Diabetol, 2012; 11: 140.

19. Krysiak R, Gdula-Dymek A, Okopien B. The effect of bezafibrate and omega-3 fatty acids on lymphocyte cytokine release and systemic inflammation in patients with isolated hypertriglyceridemia. Eur J Clin Pharmacol, 2011; 67: 1109-1117.

20. Tenenbaum A, Medvedofsky D, Fisman EZ et al. Cardiovascular events in patients received combined fibrate/statin treatment versus statin monotherapy: Acute Coronary Syndrome Israeli Surveys data. PLoS One, 2012; 7: e35298.

21. Hvidtfeldt UA, Rasmussen S, Grønbaek M, Becker U, Tolstrup JS. Influence of smoking and alcohol consumption on admissions and duration of hospitalization. Eur J Public Health, 2010; 20: 376-382.

22. Ezzati M, Henley SJ, Thun MJ, Lopez AD. Role of smoking in global and regional cardiovascular mortality. Circulation, 2005; 112: 489-497.

23. MacKenzie TD, Bartecchi CE, Schrier RW. The human costs of tobacco use (2). N Engl J Med, 1994; 330: 975-980.

24. Baliunas D, Patra J, Rehm J, Popova S, Taylor B. Smoking-attributable morbidity: Acute care hospital diagnoses and days of treatment in Canada, 2002. BMC Public Health, 2007; 7: 247.

25. Wu J, Sin DD. Improved patient outcome with smoking cessation: when is it too late? Int J Chron Obstruct Pulmon Dis, 2011; 6: 259-267.

26. Yanbaeva DG, Dentener MA, Creutzberg EC, Wesseling G, Wouters EF. Systemic effects of smoking. Chest, 2007; 131: 1557-1566.

27. Aksoy S, Cam N, Gurkan U et al. Oxidative stress and severity of coronary artery disease in young smokers with acute myocardial infarction. Cardiol J, 2012; 19: 381-386.

28. Leone A. Smoking, haemostatic factors, and cardiovascular risk. Curr Pharm Des, 2007; 13: 1661-1667.

29. Takada JY, Ramos RB, Roza LC, Avakian SD, Ramires JA, Mansur A de P. In-hospital death in acute coronary syndrome was related to admission glucose in men but not in women. Cardiovasc Diabetol, 2012; 11: 47. 